\title{
Urinary ATP may be a biomarker for bladder outlet obstruction and its severity in patients with benign prostatic hyperplasia
}

\author{
Zhenghao Chen ${ }^{1}$, Yaxiao Liu ${ }^{2}$, Mengmeng Zhao ${ }^{1}$, Shulu $\mathrm{Zu}^{1}$, Yan $\mathrm{Li}^{2}$, Benkang Shi' ${ }^{2}$ Shaoyong Wang ${ }^{1}$, \\ Xiulin Zhang ${ }^{1}$ \\ ${ }^{1}$ Department of Urology, Second Hospital of Shandong University, Jinan 250000, China; ${ }^{2}$ Department of Urology, Qilu Hospital of Shandong \\ University, Jinan 250000, China \\ Contributions: (I) Conception and design: X Zhang, S Wang, B Shi; (II) Administrative support: Z Chen; (III) Provision of study materials or patients: \\ Z Chen, Y Liu, M Zhao; (IV) Collection and assembly of data: Z Chen, M Zhao, Y Li; (V) Data analysis and interpretation: S Zu, B Shi, Z Chen, X \\ Zhang; (VI) Manuscript writing: All authors; (VII) Final approval of manuscript: All authors. \\ Correspondence to: Xiulin Zhang; Shaoyong Wang, Department of Urology, Second Hospital of Shandong University, Jinan 250000, China. \\ Email: zhangxiulin1965@163.com; sdeywangsy@sina.com.
}

Background: Urothelial cells release ATP into the urine in response to bladder stretch. Urinary ATP concentration in benign prostatic hyperplasia $(\mathrm{BPH})$ patients was higher compared with asymptomatic controls. In this study, we aimed to explore the possibility that the urinary ATP level could be a non-invasive biomarker for bladder outlet obstruction (BOO) and its severity in BPH patients.

Methods: We included $117 \mathrm{BPH}$ patients who underwent urodynamic studies and 109 asymptomatic controls. Urine samples at normal desire (from patients and controls), instilled fluids at maximum cystometric capacity (capacity fluid), and voided fluids during a pressure-flow study (only from patients) were collected. The ATP concentration in collected samples was measured using a luciferin-luciferase bioluminescence assay and normalized to urine creatinine (ATP/Cr). The degree of BOO was quantified using the BOO index (BOOI). Correlation between urodynamic parameters and urinary ATP concentration was analyzed in BPH patients.

Results: Urinary ATP concentration of BPH patients was significantly higher compared with controls $(\mathrm{P}<0.001)$. For BPH patients, a significant positive correlation was found between urinary ATP concentration and BOOI $(\mathrm{P}<0.0001)$. Although BPH patients with detrusor overactivity or a history of acute urinary retention had increased urinary ATP, a significant positive correlation between ATP and BOOI was also observed in these patients. When BOOI $>40$ was set as a cutoff point to differentiate BOO from non-BOO patients, the area under the receiver operating characteristic (ROC) curve was $0.77(\mathrm{P}<0.001)$.

Conclusions: BPH patients with BOO released higher amounts of ATP into the urine. Urinary ATP can be used as a non-invasive biomarker of BOO, and its level may also have a predictive value for the degree of obstruction.

Keywords: Lower urinary tract symptoms; benign prostatic hyperplasia (BPH); adenosine triphosphate; bladder outflow obstruction (BOO)

Submitted Nov 04, 2019. Accepted for publication Feb 14, 2020.

doi: $10.21037 /$ tau.2020.02.18

View this article at: http://dx.doi.org/10.21037/tau.2020.02.18

\section{Introduction}

Benign prostatic hyperplasia (BPH) occurs commonly in older men, with historical reports of up to $50 \%$ prevalence in men over the age of 50 years (1). An enlarged prostate may anatomically compress the urethra, causing bladder outlet obstruction (BOO). This results in lower urinary tract symptoms (LUTS), which include both voiding and storage symptoms (2). However, studies indicated that the 
International Prostate Symptom Scores (IPSS) might not be helpful for diagnosing patients with $\mathrm{BOO}$ and predicting its severity $(3,4)$.

A urodynamic study (UDS) is the gold standard to evaluate $\mathrm{BOO}$ and the degree of obstruction (5). However, USD is time-consuming and invasive, and it can be expensive for some patients. Additionally, some hospitals may not be able to afford the urodynamic equipment. All of these reasons require a non-invasive and less expensive test or a biomarker to evaluate the existence of $\mathrm{BOO}$ and the degree of obstruction in $\mathrm{BPH}$ patients.

Traditionally, the urinary bladder urothelium was considered to be a passive membrane, but it has been reported to have sensory neuronal-like properties and it responds to mechanical and chemical stimuli by releasing transmitters or mediators including ATP, ACh, and PGE2 (6,7). Released ATP will activate P2X3 receptors on the suburothelial afferents to trigger the micturition reflex $(8,9)$. Under some pathological conditions, the urothelium releases more ATP into the lumen, and the urinary ATP concentration was found to be increased in patients with overactive bladder (10-12), interstitial cystitis (13), and bladder infection or inflammation (14). Thus, urinary ATP was suggested as a biomarker for these situations.

In addition to the stretch stimulation during bladder filling, intravesical pressure during bladder isometric contraction could further stimulate ATP release from the urothelium (15). In support of this idea, the urinary ATP concentration in $\mathrm{BPH}$ patients has been found to be higher than asymptomatic controls and urinary ATP has been suggested to be a non-invasive biomarker of BOO (15). However, because urodynamic tests were not conducted in that study, the authors could not validate the existence of BOO, and thus, the diagnostic value of urinary ATP for BOO could not be verified.

For BPH patients in the compensated stage, the intravesical pressure during voiding would increase as the degree of BOO increases and more ATP may be released. Therefore, the degree of $\mathrm{BOO}$ might be inferred by measuring the urinary ATP concentration. In this study, the degree of $\mathrm{BOO}$ was quantified using the $\mathrm{BOO}$ index (BOOI) in $\mathrm{BPH}$ patients who underwent urodynamic exams. We also investigated the possibility that urinary ATP could be a potential non-invasive biomarker of $\mathrm{BOO}$ and the degree of BOO by analyzing the correlation between BOOI and urinary ATP.

\section{Methods}

\section{Patients}

All procedures in this study were approved by the Ethics Committees at the Second Hospital of Shandong University as well as Qilu Hospital of Shandong University (Approval number: KYU-2019(LW)018). All patients provided written informed consent before urodynamic examinations, which included consent to use their biological material. BPH patients submitted to urodynamic assessments were randomly recruited between April 2018 and May 2019. The criteria for selecting BPH patients to assess UDS were as follows: male $\geq 50$ years of age; diagnosed with symptomatic BPH; IPSS $\geq 13$; maximum urinary flow rate $\leq 12 \mathrm{~mL} / \mathrm{s}$ on a voided volume of $\geq 150 \mathrm{~mL}$; prostate volume $\geq 30 \mathrm{~mL}$ that was estimated by ultrasound, or with a history of acute urinary retention (AUR). Exclusion criteria were any with a history of malignancy of any sort (including bladder and/or prostate cancer), pelvic radiotherapy, neurologic disease, any systemic or inflammatory condition, active urinary tract infections, or renal impairment (chronic kidney disease stage $>2$ ). Initially, $150 \mathrm{BPH}$ patients were recruited, after exclusion of 33 patients, the final number was left 117 (Figure 1), the age of these BPH patients is $68.93 \pm 0.63$ years (range, $51-84$ years). One hundred and nine asymptomatic male volunteers (48-75 years of age; mean, $65.33 \pm 0.61$ years) were recruited from the Health Exam Center at the Second Hospital of Shandong University. Exclusion criteria for volunteers were any urinary tract infection, malignancy, or neurological disease (e.g., diabetes mellitus, prolapsed of intravertebral discs, and spinal trauma). All BPH patients underwent a UDS to differentiate BOO from detrusor underactivity (DU, n=24, Figure 1). For patients with AUR, 10-14 days were given to allow bladder function to recover, and these patients could void as usual when urodynamic test was conducted.

\section{Free uroflowmetry measurement}

Asymptomatic volunteers were asked to void in accordance with their normal desire into a sterile cup for ATP and creatinine $(\mathrm{Cr})$ determination. BPH patients were asked to void comfortably in accordance with their normal desire in a standing position into a urinary flowmeter. Voided volume was recorded. Midstream urine was taken for ATP and $\mathrm{Cr}$ measurement. 


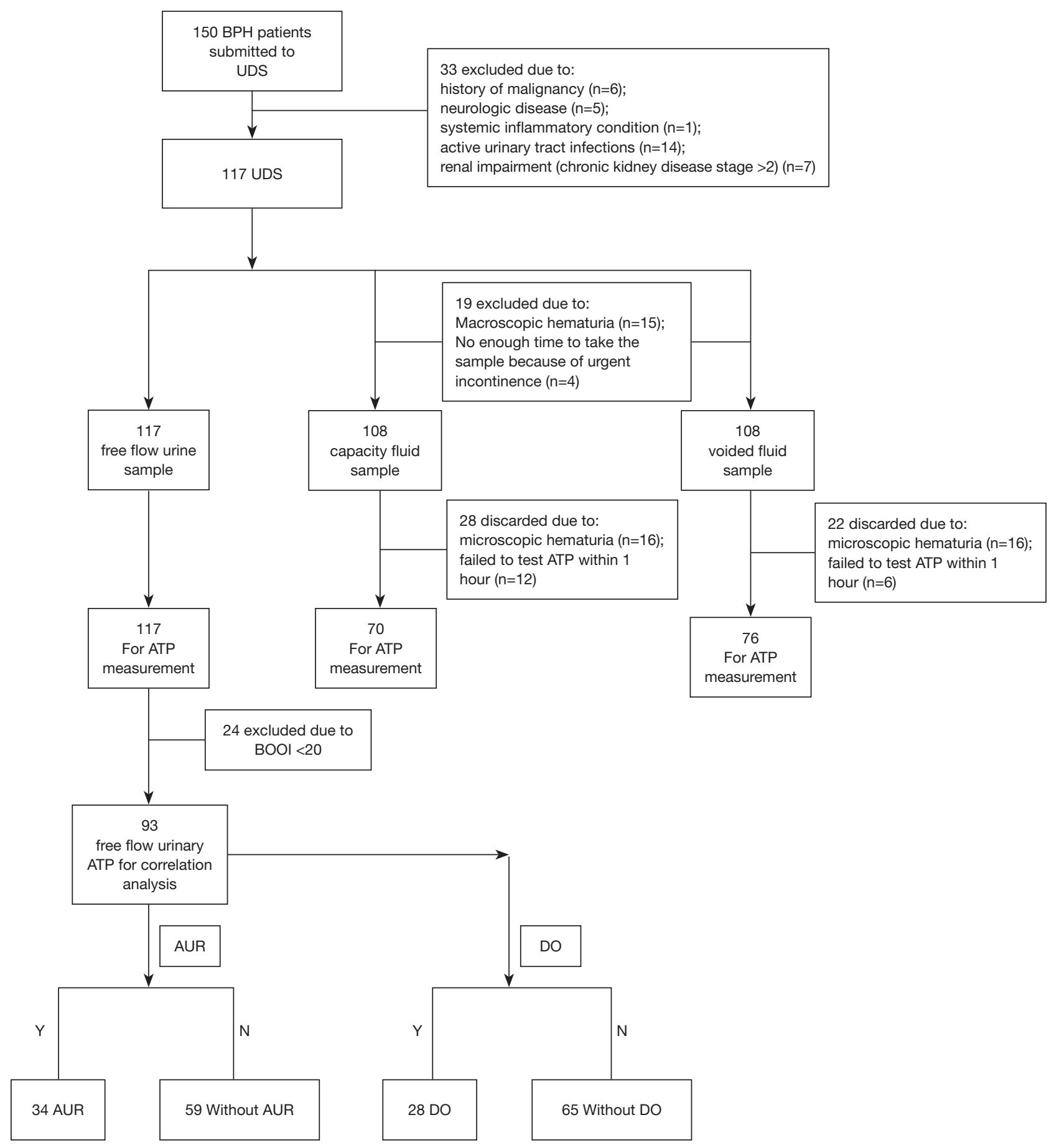

Figure 1 Flowchart of patients' selection and grouping. BPH, benign prostatic hyperplasia; BOOI, BOO index; AUR, acute urinary retention; DO, detrusor overactivity.

\section{Pressure-flow assessment}

$\mathrm{BPH}$ patients but not asymptomatic volunteers received this assessment. Computer-urodynamic investigation was conducted by one experienced investigator in accordance with the Good Urodynamic Practices Standards (16). Immediately after the free uroflowmetry measurement, the 
patient was placed into a semi-reclining position, and a $6-\mathrm{Fr}$ transurethral double-lumen catheter was inserted into their bladder to determine the postvoid residual volume (PVR). The bladder was then filled with room temperature $\left(25^{\circ} \mathrm{C}\right)$ sterile normal saline at a rate of $30-40 \mathrm{~mL} / \mathrm{min}$, and the intravesical pressure was measured. To measure detrusor pressure (Pdet), a 10-F single lumen catheter was inserted into the rectum to measure intra-abdominal pressure. The presence of detrusor overactivity (DO) was identified when Pdet spontaneously increased during the filling phase. When the maximum bladder volume was reached, perfusion was stopped, and an instilled fluid sample was taken from the bladder lumen via a perfusion catheter (this was called the capacity fluid). The patient was asked to void and pressure-flow measurements were recorded. The midstream voided fluid was sampled.

\section{Urodynamic parameters analysis}

For free uroflowmetry analysis, artifacts need to be manually corrected and the maximum value was selected for maximum urinary flow rate (Qmax). Parameters analyzed include Qmax, voided volume (VV), and PVR.

For pressure-flow parameter analysis, only measurements without straining were considered. Analyzed parameters include maximum Pdet (MaxPdet), Pdet at Qmax (Pdet@ Qmax), and bladder obstruction index (BOOI = Pdet@ Qmax - 2Qmax). In addition to BOOI, the degree of BOO was also classified into six grades (from 0 to VI) using a Schaefer nomogram.

\section{Measurement of urinary ATP and creatinine}

ATP concentrations in urine samples at the normal desire from control or $\mathrm{BPH}$ patients as well as in instilled fluids that were taken during the pressure-flow study for $\mathrm{BPH}$ patients. These samples were measured immediately (within 1 hour) after they were taken, otherwise, it was discarded (Figure 1). Samples were first centrifuged (500 rpm for $5 \mathrm{~min}$ ) to eliminate dead cells and determine whether microscopic hematuria was present, and then the supernatant was divided into two equal parts, as follows: one for the ATP test and the other for creatinine concentration measurement. Measurement of lactate dehydrogenase (LDH) level, which is a fairly stable intracellular enzyme that is widely used as an indicator of cell integrity revealed no more cell damage after centrifugation. Urinary ATP values were normalized to $\mathrm{Cr}$ to limit sample variation or to eliminate the influence of the kidney on ATP levels. The person who analyzed the ATP measurement was blinded to the sample source.

Urine or instilled fluid samples $(100 \mu \mathrm{L})$ were added to a mixture of luciferin-luciferase $(100 \mu \mathrm{L})$, in accordance with the manufacturer's instructions using the Promega CellTiterGlo $^{\mathrm{TM}}$ Luminescent Cell Viability Assay Kit (Promega). ATP was measured using the GloMax ${ }^{\mathrm{TM}}$ 20/20 luminometer (Promega). Sample bioluminescence was compared with that of standard amounts of ATP that were used in the same concentration range. Standard ATP samples were prepared daily, and all samples were run in duplicate.

The urinary creatinine level was measured using a standard commercial kit (Jiancheng Bioengineering Institute, Nanjing, China) with a spectrophotometer (INFINITE M200PRO, Tecan, China).

\section{Statistical analysis}

Statistical analyses were performed using SigmaPlot 10.0 (Systat Software Inc.). Results are reported as the mean \pm standard error (SE). The Kolmogorov-Smirnov test was used to check for normality. An unpaired Student's $t$-test was used for statistical analysis between groups. For multiple group comparisons, a one-way ANOVA test with Dunn's post-test modification was used. Correlation between variables was analyzed using the Pearson or Spearman test, and further correlation between variables was analyzed using linear regression analysis. $\mathrm{P}<0.05$ (twotailed) values were considered to be statistically significant. The diagnostic potential of urinary ATP was assessed using receiver operating characteristic (ROC) plots and the area under the curve (AUC).

\section{Results}

\section{Urinary ATP in BPH patients was higher compared with asymptomatic controls}

\section{Comparison of urinary ATP concentration}

The clinical characteristics of BPH patients $(n=117)$ and male asymptomatic controls $(\mathrm{n}=109)$ were listed in Table 1$)$, There was no significant difference in age between the control and $\mathrm{BPH}$ patients $(65.33 \pm 0.61$ vs. $68.93 \pm 0.63$ years, $\mathrm{P}>0.05)$. BPH patients had significant higher IPSS $(21.33 \pm 1.85)$ than asymptomatic volunteers $(3.53 \pm 0.7$, $\mathrm{P}<0.001)$. Urinary ATP concentration in $\mathrm{BPH}$ patients $(118.54 \pm 7.18 \mathrm{nM})$ was significantly higher compared with normal controls $(47.67 \pm 2.42 \mathrm{nM}$; Figure $2 A)$. This was also 
Table 1 Characteristics of BPH patients and asymptomatic controls

\begin{tabular}{lccc}
\hline Parameters & Patients, $\mathrm{n}=117$, mean \pm SD & Control, $\mathrm{n}=109$, mean \pm SD & P value \\
\hline Age & $68.93 \pm 0.63$ & $65.33 \pm 0.61$ & $>0.050$ \\
IPSS & $21.33 \pm 1.85$ & $3.53 \pm 0.70$ & $<.001$ \\
PV $(\mathrm{cc})$ & $75.58 \pm 13.63$ & - & - \\
Qmax $(\mathrm{mL} / \mathrm{s})$ & $8.13 \pm 0.47$ & - & - \\
PVR $(\mathrm{mL})$ & $22.07 \pm 9.47$ & $47.67 \pm 2.42$ & $<0.001$ \\
Urinary ATP $(\mathrm{nM})$ & $118.54 \pm 7.18$ & $93.28 \pm 13.85$ & $>0.050$ \\
Cr $(\mathrm{mg} / \mathrm{dL})$ & $95.49 \pm 9.92$ & $51.80 \pm 2.63$ & $<0.001$ \\
ATP/Cr $(\mathrm{pmol} / \mathrm{mg})$ & $126.20 \pm 7.99$ & & - \\
\hline
\end{tabular}

IPSS, the international Prostate Symptom Scores; PV, prostate volume; Qmax, maximum urinary flow rate; PVR, postvoid residual volume; Cr, creatinine.

A

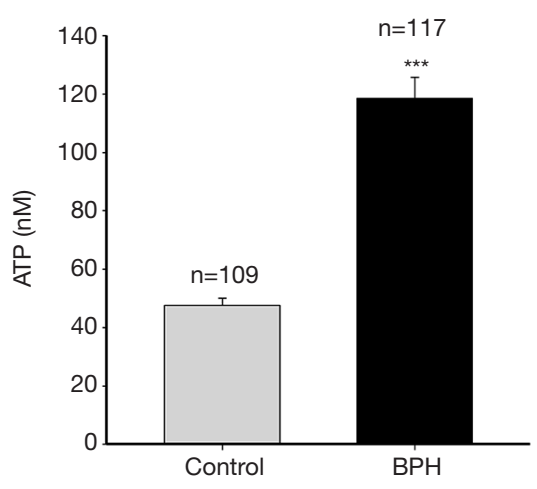

B

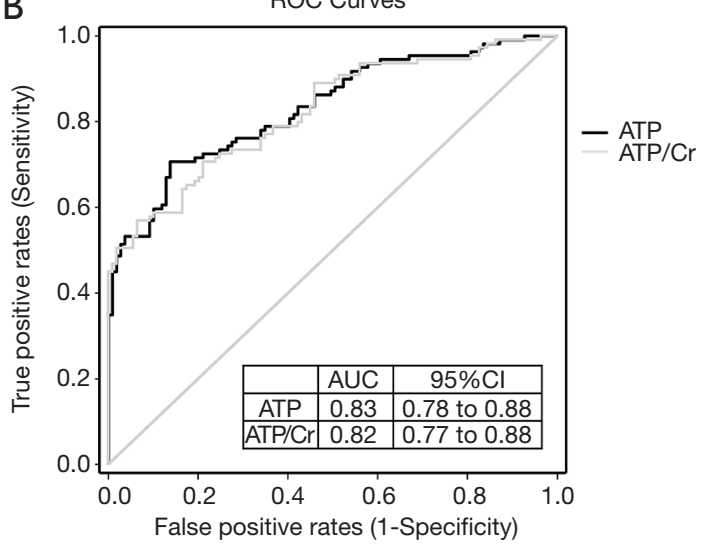

Figure 2 The diagnostic value of urinary ATP to distinguish between BPH patients and normal people. (A) Comparison of urinary ATP concentration between BPH patients and asymptomatic controls. There was a significant difference between the two groups $\left.{ }^{* * *}, \mathrm{P}<0.001\right)$. (B) ROC curves of urinary ATP concentration. ATP (black line) and the results corrected for urinary creatinine levels (ATP/Cr, grey line) were plotted as continuous variables to distinguish between true-positive and false-positive rates among BPH patients and controls. AUC was 0.83 for ATP and 0.82 for ATP/Cr, and both results were significant $(\mathrm{P}<0.001)$. There were no differences $(\mathrm{P}>0.05)$ between the two variables (ATP vs. ATP/Cr). BPH, benign prostatic hyperplasia; ROC, receiver operating characteristic; AUC, area under the curve.

true when urinary ATP was normalized to urine creatinine levels (ATP/Cr, $126.20 \pm 7.99$ vs. $51.8 \pm 2.63 \mathrm{pmol} / \mathrm{mg}$, $\mathrm{P}<0.0001)$. There was no significant difference in the urinary creatinine level between the two groups $(95.49 \pm 9.92$ vs. $93.28 \pm 13.85 \mathrm{mg} / \mathrm{dL}, \mathrm{P}>0.05)$. Thus, unless stated, we used the ATP concentration (nM) without normalization for most of the following analyses.

\section{ROC curve}

To assess the diagnostic value of urinary ATP for BPH, the ROC curves (Figure $2 B$ ) for urinary ATP concentration
(ATP, black line) and ATP/Cr (grey line) were plotted for all $\mathrm{BPH}$ patients and controls. The AUC value was 0.83 for ATP (95\% CI: $0.78-0.88, \mathrm{P}<0.001)$ and 0.82 for ATP/ Cr (95\% CI: 0.77-0.88, $\mathrm{P}<0.001)$. There was no difference between the two variables $(\mathrm{P}>0.05)$.

\section{Correlation of urinary ATP with urodynamic parameters in BPH patients}

ATP concentration in urine and urodynamic fluids For BPH patients, ATP concentrations in urine taken 
Table 2 Correlation of urinary ATP with age and urodynamic parameters in BPH patients

\begin{tabular}{|c|c|c|c|c|}
\hline Parameters & Mean & Std. Error & Coefficient & $P$ value \\
\hline \multicolumn{5}{|l|}{ Free uroflowmetry } \\
\hline Qmax (mL/s) & 8.134 & 0.466 & -0.177 & 0.123 \\
\hline $\mathrm{VV}(\mathrm{mL})$ & 180.128 & 12.101 & -0.276 & 0.006 \\
\hline \multicolumn{5}{|l|}{ Pressure-flow } \\
\hline Pdet@Qmax & 75.264 & 2.787 & 0.379 & $<0.001$ \\
\hline MaxPdet & 80.949 & 3.116 & 0.301 & 0.002 \\
\hline BOOI & 64.059 & 2.510 & 0.566 & $<0.001$ \\
\hline
\end{tabular}

Analysis was based on $93 \mathrm{BPH}$ patients with $\mathrm{BOOI}>20$. BPH, benign prostatic hyperplasia; Qmax, maximum urinary flow rate; VV, voided volume; PVR, postvoid residual volume; Pdet@Qmax, Pdet at Qmax; MaxPdet, maximum Pdet; BOOI, bladder obstruction index (BOOI = Pdet@Qmax - 2Qmax).

at free uroflow (free flow urine), instilled fluids taken at maximum bladder volume (capacity fluid), and fluids taken at voiding during the pressure-flow study (voided fluid) were measured. The ATP concentration in free-flow urine $(118.54 \pm 7.18 \mathrm{nM}, \mathrm{n}=117)$ was not significantly different from that of the capacity fluid $(109.99 \pm 12.45 \mathrm{nM}, \mathrm{n}=70$, $\mathrm{P}>0.05)$ or from that of the voided fluid $(135.26 \pm 14.64 \mathrm{nM}$, $\mathrm{n}=76$, one-way ANOVA, $\mathrm{P}>0.05)$. The ATP concentration in voided fluid tended to be higher compared with the capacity fluid, but it did not reach significance $(\mathrm{P}>0.05)$.

\section{Correlation with urodynamic parameters}

Based on the results above, the ATP concentration in free uroflow urine was used in the following analysis to explore the potential correlation with urodynamic parameters for BPH patients with BOOI $>20(n=93)$. Pearson correlation analysis was performed, and analysis results were summarized in Table 2. Void volume (VV, $180.1 \pm 12.1 \mathrm{~mL}$ ) in $\mathrm{BPH}$ patients showed an inverse correlation with urinary ATP levels $(\mathrm{P}=0.006)$, while the BOOI $(64.06 \pm 2.51$, $\mathrm{P}<0.001)$, Pdet@Qmax $\left(75.26 \pm 2.79 \mathrm{cmH}_{2} \mathrm{O}, \mathrm{P}<0.001\right)$, and MaxPdet $\left(80.95 \pm 3.12 \mathrm{cmH}_{2} \mathrm{O}, \mathrm{P}=0.002\right)$ showed a positive correlation with the urinary ATP. Qmax $(8.13 \pm 0.47 \mathrm{~mL} / \mathrm{s})$ tended to be correlated with urinary ATP, but it did not reach significance $(P>0.05)$. Age and $P V R$ showed no correlation.

\section{Linear regression analysis of urinary ATP with BOOI}

To examine the possibility of urinary ATP as a non-invasive biomarker for the severity of BOO, further correlation tests were performed (Figure 3). As expected, linear regression analysis showed a significant positive correlation between BOOI and urinary ATP in BPH patients with BOOI $>20$ $(\mathrm{r}=0.5441, \mathrm{P}<0.0001$; Figure $3 A)$.

To examine the diagnostic value of urinary ATP for BOO, BOOI $>40$ was set as a cutoff point to differentiate BOO from non-BOO patients $(16,17)$. ROC curves showed a high AUC for both ATP and ATP/Cr (Figure 3B), which was 0.77 (95\% CI: $0.67-0.87)$ for ATP and 0.76 (95\% CI: 0.66-0.87) for ATP/Cr. Both were statistically significant $(\mathrm{P}<0.001)$.

\section{Situations that influence the urinary ATP level and its correlation with BOOI}

\section{Urinary ATP in patients with DO or a history of AUR}

Situations such as a history of AUR or the presence of DO may influence the urinary ATP concentration (10-12). In this study, the urinary ATP concentration of BPH patients with DO $(169.38 \pm 20.64 \mathrm{nM}, \mathrm{n}=28)$ was significantly higher compared with patients without DO $(114.46 \pm 8.75 \mathrm{nM}, \mathrm{n}=65, \mathrm{P}=0.006$; Figure $4 A)$; it was also significantly higher in patients with a history of AUR $(162.35 \pm 18.47 \mathrm{nM}, \mathrm{n}=34)$ compared with those without AUR $(112.92 \pm 8.79 \mathrm{nM}, \mathrm{n}=59, \mathrm{P}=0.006$; Figure $4 B)$. To note, AUR patients have a significantly larger BOOI than patients without AUR (162.35 \pm 18.47 vs. $112.92 \pm 8.79$, $\mathrm{P}=0.019)$. 

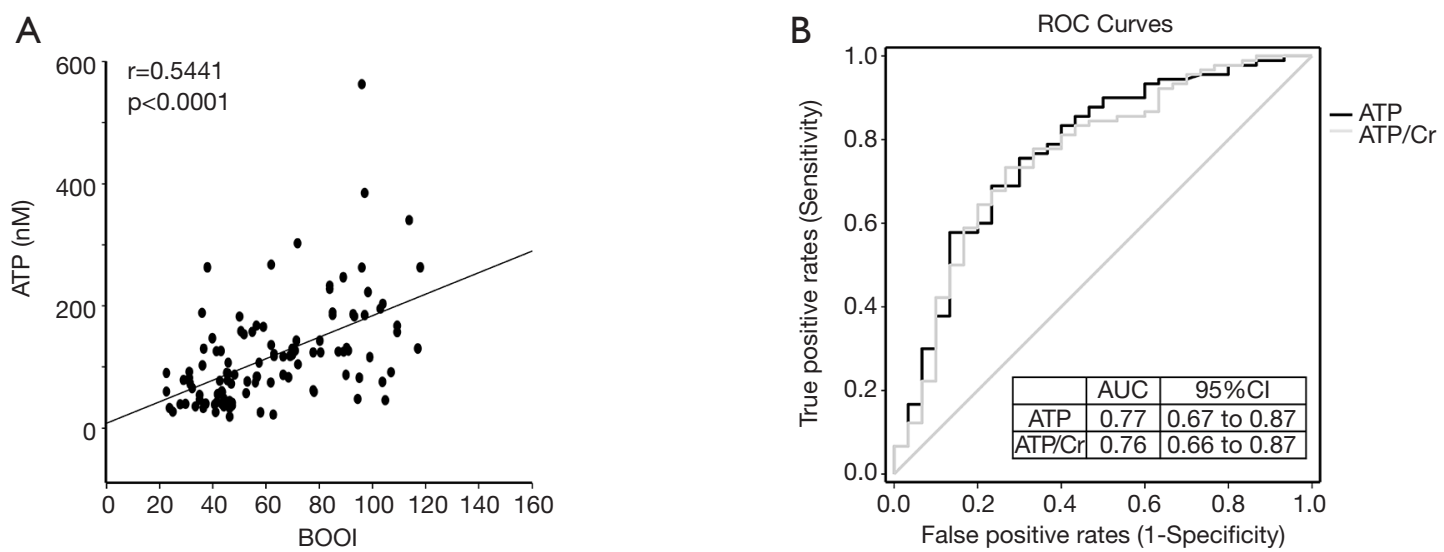

Figure 3 The diagnostic value of urinary ATP for BOO. (A) Linear regression analysis of urinary ATP concentration with BOOI for BPH patients. There was a significant positive correlation of urinary ATP with BOOI $(r=0.5441, \mathrm{P}<0.0001)$ for all $\mathrm{BPH}$ patients. (B) ROC curves of urinary ATP concentration among BPH patients. With the cut-off value of BOOI at 40, BPH patients were divided into two groups: with BOO (BOOI >40) and without BOO (BOOI <40). ATP (black line) and ATP/Cr (grey line) were plotted as continuous variables to distinguish between true-positive and false-positive rates. The AUC was 0.77 for ATP and 0.76 for ATP/Cr, which were both statistically significant $(\mathrm{P}<0.001)$. ROC, receiver operating characteristic; AUC, area under the curve; BOOI, BOO index; BPH, benign prostatic hyperplasia.

\section{Impact of DO and AUR on the correlation}

Despite the influence of DO or AUR on urinary ATP (Figure $4 A, B$ ), when the linear regression analysis was performed separately (Figure $4 C, D, E, F$ ), there was a significant positive correlation between urinary ATP and BOOI either in patients with DO $(\mathrm{r}=0.4413, \mathrm{P}=0.0187$, Figure $4 C)$ or without DO $(\mathrm{r}=0.5965, \mathrm{P}<0.0001$, Figure $4 E)$ and in patients with AUR $(\mathrm{r}=0.3813, \mathrm{P}=0.0261$, Figure $4 D)$ or without AUR (r=0.5688, $\mathrm{P}<0.0001$, Figure 4F).

\section{Discussion}

In this study, we confirmed the finding that urinary ATP is higher in $\mathrm{BPH}$ patients compared with asymptomatic controls (15). We also quantified the degree of BOO using the BOOI in $\mathrm{BPH}$ patients who underwent urodynamic studies, and we found that the urinary ATP concentration was positively correlated with BOOI. Moreover, this correlation was not affected by the presence of DO or a history of AUR. When BOOI $>40$ was set as a cutoff point to differentiate $\mathrm{BOO}$ from NO-BOO patients, a high area under the ROC curve ( 0.77 for ATP; 0.76 for ATP/Cr) was noted. Our results suggest that the urinary ATP level in $\mathrm{BPH}$ patients might be used as a non-invasive biomarker for BOO as well as its severity.

Although it is hard to confirm the origin of urinary ATP, most studies have suggested that it is mainly from the urothelium (18). We and other researchers (15) propose that in addition to stretch-induced ATP release during filling, increased wall tension produced by intravesical pressure would further enhance ATP release from the urothelium. This view could be supported by the following evidence from us and others: (I) ATP concentrations in voided fluids tend to be higher compared with capacity fluids; (II) a significant positive correlation was found between MaxPdet or Pdet@Qmax and the ATP level as well as between the BOOI and the ATP level (Table 2); (III) urinary ATP concentration in BPH patients was higher compared with normal controls (15) and urothelium strips from $\mathrm{BPH}$ patients released five-times more ATP compared with preparations from normal controls (19); and (IV) in $\mathrm{BPH}$ patients, after treatment with an alpha-adrenergic receptor antagonist to reduce urethral resistance, the urinary ATP level was decreased accordingly (19). In addition to increased ATP release from the urothelium (20), decreased ATP catabolism (21), enhanced ATP-induced ATP release (13), and increased ATP release from parasympathetic nerves (22). All these factors occurred in the BPH bladder and they may also contribute to the higher urinary ATP concentration.

Consistent with Silva-Ramos's study (15), we found that urinary ATP concentration in $\mathrm{BPH}$ patients was significantly higher compared with asymptomatic controls (Figure $2 A$ ). Additionally, also consistent with their study, ROC curves showed a large AUC for ATP and ATP/ 
A

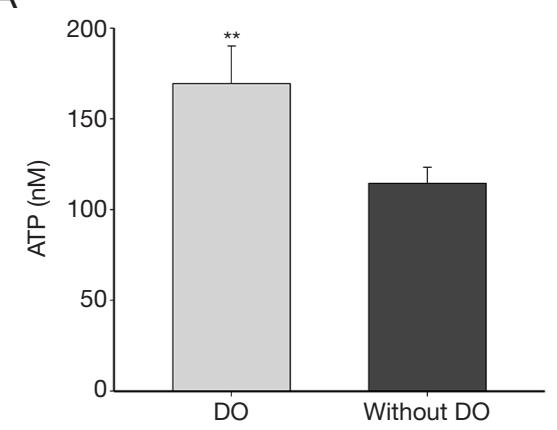

C

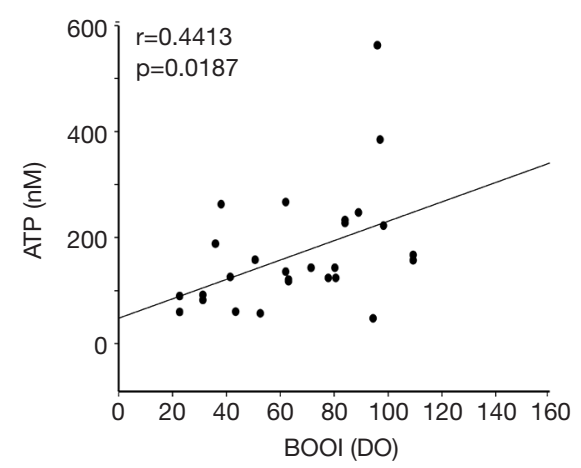

E

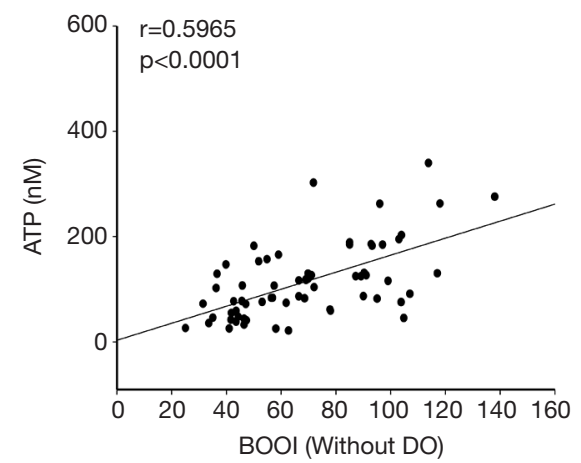

B

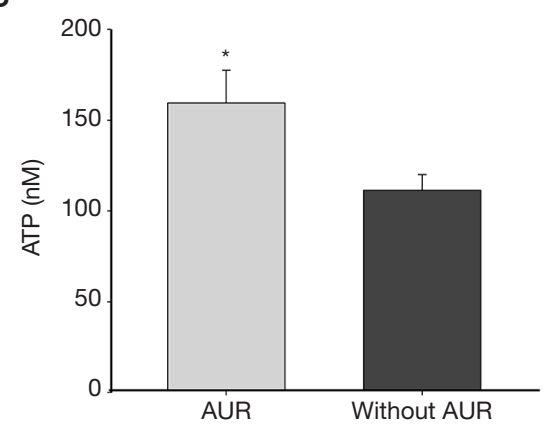

D

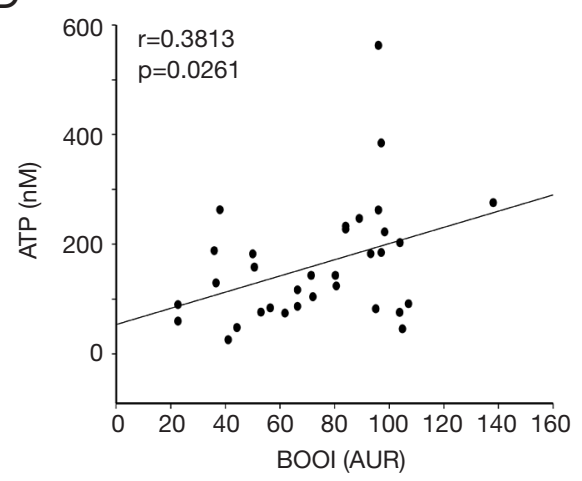

$\mathrm{F}$

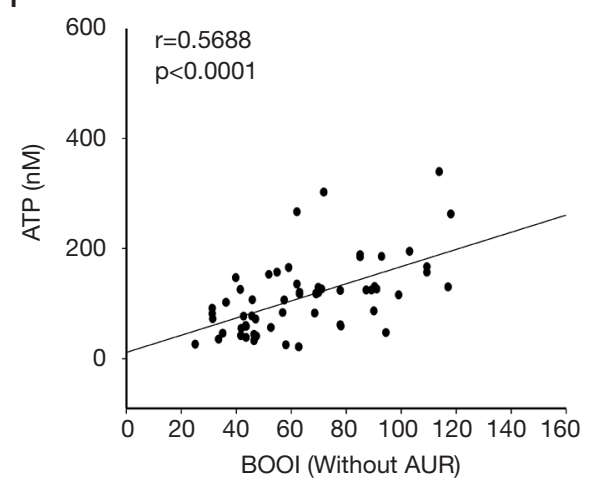

Figure 4 Urinary ATP concentration in BPH patients with and without DO, or with and without AUR $(A, B)$ and its further correlation with BOOI. (A) The urinary ATP concentration was higher in BPH patients with DO compared with those without DO ( $\mathrm{P}=0.006)$, (B) it was significantly higher in $\mathrm{BPH}$ patients with a history of AUR compared with those without AUR $(\mathrm{P}=0.019)$. *, $\mathrm{P}<0.05$; **, $\mathrm{P}<0.01$. There was a significant positive correlation of urinary ATP with BOOI for BPH patients with DO (C) or without DO (E). A significant positive correlation was also present when the analysis was performed separately in patients with AUR (D) and without AUR (F). BPH, benign prostatic hyperplasia; DO, detrusor overactivity; AUR, acute urinary retention; BOOI, BOO index.

Cr (Figure 2B), which were both significantly different $(\mathrm{P}<0.001)$. This suggests that urinary ATP is an excellent factor to use to distinguish between asymptomatic controls and BPH patients.
In our study, we found that the free uroflow urinary ATP concentration was similar to that in urodynamic fluids. This result establishes that free uroflow urinary ATP could be a good alternative biomarker when urodynamic exams are not 
available. However, we found that the ATP concentration in voided fluid during the pressure-flow test tends to be higher compared with capacity fluids, suggesting that increased Pdet during micturition would further increase the ATP release.

In this study, no significant correlation was found between age and urinary ATP concentration for BPH patients (Table 2) or for normal subjects (data not shown). In terms of the age effect, some reports showed that urinary ATP increases with age (23), while others found a negative correlation $(15)$ or no age effect $(14,24)$. The reasons for these inconsistent results remain unclear.

Consistent with Silva-Ramos's study (15), there was a non-significant trend toward an inverse correlation between Qmax and urinary ATP in our study. Additionally, in agreement with their study, we did not find a correlation between PVR and urinary ATP. However, in contrast to their report that $\mathrm{VV}$ has a positive correlation with ATP, we found a significant inverse correlation of VV with ATP (Table 2). A significant inverse correlation of ATP with the maximum bladder capacity has was found in normal women and women with overactive bladder $(\mathrm{OAB})(11,12)$. The reasons for this inconsistency between the studies remains unclear. One reason may come from the different subpopulations between Silva-Ramos's study and our study. Compared with patients in their study, our patients had more severe LUTS, for example, IPSS of our patients is larger than theirs $(21.33 \pm 1.85$ vs. $15.07 \pm 0.73)$, we had more patients with a history of AUR (34 of 117). We suggest that two opposite effects of $\mathrm{VV}$ or bladder volume on ATP concentration should be considered. On the one hand, a larger bladder capacity would enhance more ATP release by producing a greater extent of stretch, while on the other hand, a larger bladder volume may have a dilution effect on ATP.

The presence of DO during bladder filling would evoke an urgency sensation and OAB symptoms. Several studies indicated that urinary ATP is increased in OAB patients with DO, which is either neurogenic or idiopathic $(10,12,25)$, and thus, urinary ATP may be a biomarker for DO. In our study, the incidence of DO in $\mathrm{BPH}$ patients was $24 \%$ (28/117). This is consistent with published results $(10,12,25)$, and the urinary ATP concentration was higher in our BPH patients with DO compared with those without DO (Figure 4A).

$\mathrm{BPH}$ patients may progress to AUR in the late stages. However, AUR patients who were included in our study could void during the pressure-flow study and during the free uroflowmetry test after 10-14 days of recovery. We found that the urinary ATP concentration was higher in $\mathrm{BPH}$ patients who had a history of AUR compared with non-AUR patients. The explanation for this remains unclear. A higher degree of BOO in AUR patients might explain the difference. Additionally, urinary catheterinduced irritation might not be the cause. In three AUR patients, we collected urine samples on 5 consecutive days from the urinary catheter, and no significant differences were found among the ATP concentrations on these 5 days (data not shown).

The most important finding in our study was that urinary ATP concentration is positively correlated with BOOI. BOOI is a continuous variable, and it is usually used to quantify the degree of BOO (26). Based on the BOOI value, the patients were classified into obstructed (BOOI $>40$ ), equivocal (BOOI 20-40), and unobstructed (<20) (27). In some reports, equivocal (BOOI 20-40) has been considered to be obstructed $(28,29)$. Thus, to fully evaluate the correlation of urinary ATP with BOOI, we included BPH patients with equivocal BOOI scores (Figures $3 A$ and 4 ). However, when the diagnostic value of urinary ATP for BOO was determined using ROC curves (Figure $3 B$ ), only the obstructed $\mathrm{BPH}$ patients were selected (BOOI $>40$ ).

In addition to BOOI, two other voiding parameters (MaxPdet and Pdet@Qmax) were also found to be positively correlated with urinary ATP (Table 2). Our findings showed that, besides stretching during bladder filling, increased intravesical pressure during voiding is another stimulus that promotes further urothelial ATP release. These findings are in contrast to one study in healthy women or OAB patients that showed no correlation between Pdet and urinary ATP (11). In that study, OAB patients who had Pdet > $60 \mathrm{cmH}_{2} \mathrm{O}$ were excluded, and thus, it is impossible to obtain the complete picture of Pdet correlation with ATP. The inconsistent reports might also indicate that there is a sex difference.

Patients with BPH released higher amounts of ATP into the urine, and the amount increased with the increasing degree of BOO. A significant positive correlation of urinary ATP with BOOI and the high AUC of the ROC curve indicated the value of urinary ATP as a non-invasive biomarker to identify $\mathrm{BOO}$ and to predict its severity in $\mathrm{BPH}$ patients. Compared with the invasive and expensive features of USD, urinary ATP measurement is simple, non-invasive, inexpensive, and reliable, and it might be an alternative method to evaluate BOO. 


\section{Acknowledgments}

We thank Jodi Smith, PhD, from Liwen Bianji, Edanz Editing China (www.liwenbianji.cn/ac), for editing the English text of a draft of this manuscript.

Funding: This work was supported by a grant from the National Natural Science Funds of China (81670686 \& 81470987), the Key R and D Project of Shandong Province (2017GSF18149), and the TaiShan Scholar Foundation to Benkang Shi.

\section{Footnote}

Conflicts of Interest: All authors have completed the ICMJE uniform disclosure form (available at http://dx.doi. org/10.21037/tau.2020.02.18). The authors have no conflicts of interest to declare.

Ethical Statement: The authors are accountable for all aspects of the work in ensuring that questions related to the accuracy or integrity of any part of the work are appropriately investigated and resolved. All procedures in this study were approved by the Ethics Committees at the Second Hospital of Shandong University as well as Qilu Hospital of Shandong University (Approval number: KYU2019(LW)018). All patients provided written informed consent before urodynamic examinations, which included consent to use their biological material.

Open Access Statement: This is an Open Access article distributed in accordance with the Creative Commons Attribution-NonCommercial-NoDerivs 4.0 International License (CC BY-NC-ND 4.0), which permits the noncommercial replication and distribution of the article with the strict proviso that no changes or edits are made and the original work is properly cited (including links to both the formal publication through the relevant DOI and the license). See: https://creativecommons.org/licenses/by-nc$\mathrm{nd} / 4.0 /$.

\section{References}

1. Zhang W, Zhang X, Li H, et al. Prevalence of lower urinary tract symptoms suggestive of benign prostatic hyperplasia (LUTS/BPH) in China: results from the China Health and Retirement Longitudinal Study. BMJ Open 2019;9:e022792.

2. Barry MJ, Fowler FJJ, O'Leary MP, et al. The American
Urological Association symptom index for benign prostatic hyperplasia. The Measurement Committee of the American Urological Association. J Urol 1992;148:1549-57.

3. Barry MJ, Girman CJ, O'Leary MP, et al. Using repeated measures of symptom score, uroflowmetry and prostate specific antigen in the clinical management of prostate disease. Benign Prostatic Hyperplasia Treatment Outcomes Study Group. J Urol 1995;153:99-103.

4. Steele GS, Sullivan MP, Sleep DJ, et al. Combination of symptom score, flow rate and prostate volume for predicting bladder outflow obstruction in men with lower urinary tract symptoms. J Urol 2000;164:344-8.

5. Rosier P. Contemporary diagnosis of lower urinary tract dysfunction. Version 1 F1000Res 2019;8. doi: 10.12688/ f1000research.16120.1.

6. Birder LA. Urinary bladder urothelium: molecular sensors of chemical/thermal/mechanical stimuli. Vascul Pharmacol 2006;45:221-6.

7. Merrill L, Gonzalez EJ, Girard BM, et al. Receptors, channels, and signalling in the urothelial sensory system in the bladder. Nat Rev Urol 2016;13:193-204.

8. Wang EC, Lee JM, Ruiz WG, et al. ATP and purinergic receptor-dependent membrane traffic in bladder umbrella cells. J Clin Invest 2005;115:2412-22.

9. Cockayne DA, Hamilton SG, Zhu QM, et al. Urinary bladder hyporeflexia and reduced pain-related behaviour in P2X3-deficient mice. Nature 2000;407:1011-5.

10. Cheng Y, Mansfield KJ, Allen W, et al. ATP during early bladder stretch is important for urgency in detrusor overactivity patients. Biomed Res Int 2014;2014:204604.

11. Cheng Y, Mansfield KJ, Allen W, et al. Does Adenosine Triphosphate Released Into Voided Urodynamic Fluid Contribute to Urgency Signaling in Women With Bladder Dysfunction? Journal of Urology 2010;183:1082-6.

12. Silva-Ramos M, Silva I, Oliveira O, et al. Urinary ATP may be a dynamic biomarker of detrusor overactivity in women with overactive bladder syndrome. PLoS One 2013;8:e64696.

13. Sun Y, Keay S, De Deyne PG, et al. Augmented stretch activated adenosine triphosphate release from bladder uroepithelial cells in patients with interstitial cystitis. J Urol 2001;166:1951-6.

14. Gill K, Horsley H, Kupelian AS, et al. Urinary ATP as an indicator of infection and inflammation of the urinary tract in patients with lower urinary tract symptoms. BMC Urol 2015;15:7.

15. Silva-Ramos M, Silva I, Oliveira JC, et al. Increased 
Urinary Adenosine Triphosphate in Patients With Bladder Outlet Obstruction Due to Benign Prostate Hyperplasia. The Prostate 2016;76:1353-63.

16. Arif M, Groen J, Boeve ER, et al. Noninvasive Diagnosis of Bladder Outlet Obstruction in Patients with Lower Urinary Tract Symptoms Using Ultrasound Decorrelation Analysis. J Urol 2016;196:490-7.

17. Garg G, Sankhwar SN, Goel A, et al. Evaluation of resistive index of the prostate and bladder sonomorphologic parameters as replacements for urodynamics to predict bladder outlet obstruction in patients with lower urinary tract symptoms suggestive of benign prostatic hyperplasia. Low Urin Tract Symptoms 2019;11:163-8.

18. Kumar V, Chapple CC, Chess-Williams R. Characteristics of adenosine triphosphate [corrected] release from porcine and human normal bladder. J Urol 2004;172:744-7.

19. Silva I, Ferreirinha F, Magalhães-Cardoso M, et al. Activation of P2Y6 Receptors Facilitates Nonneuronal Adenosine Triphosphate and Acetylcholine Release from Urothelium with the Lamina Propria of Men with Bladder Outlet Obstruction. J Urol 2015;194:1146-54.

20. Sun Y, MaLossi J, Jacobs SC, et al. Effect of doxazosin on stretch-activated adenosine triphosphate release in bladder urothelial cells from patients with benign prostatic hyperplasia. Urology 2002;60:351-6.

21. Harvey RA, Skennerton DE, Newgreen D, et al. The contractile potency of adenosine triphosphate and ectoadenosine triphosphatase activity in guinea pig detrusor and detrusor from patients with a stable, unstable or obstructed bladder. J Urol 2002;168:1235-9.

22. Silva-Ramos M, Silva I, Faria $M$, et al. Impairment of ATP hydrolysis decreases adenosine A1 receptor tonus favoring cholinergic nerve hyperactivity in the obstructed human

Cite this article as: Chen Z, Liu Y, Zhao M, Zu S, Li Y, Shi B, Wang S, Zhang X. Urinary ATP may be a biomarker for bladder outlet obstruction and its severity in patients with benign prostatic hyperplasia. Transl Androl Urol 2020;9(2):284294. doi: 10.21037/tau.2020.02.18 urinary bladder. Purinergic Signal 2015;11:595-606.

23. Yoshida M, Miyamae K, Iwashita H, et al. Management of detrusor dysfunction in the elderly: changes in acetylcholine and adenosine triphosphate release during aging. Urology 2004;63:17-23.

24. Sugaya K, Nishijima S, Kadekawa K, et al. Relationship between lower urinary tract symptoms and urinary ATP in patients with benign prostatic hyperplasia or overactive bladder. Biomed Res 2009;30:287-94.

25. Kumar V, Chapple CR, Rosario D, et al. In vitro release of adenosine triphosphate from the urothelium of human bladders with detrusor overactivity, both neurogenic and idiopathic. Eur Urol 2010;57:1087-92.

26. Oelke M, Rademakers KLJ, van Koeveringe GA, et al. Unravelling detrusor underactivity: Development of a bladder outlet resistance-Bladder contractility nomogram for adult male patients with lower urinary tract symptoms. Neurourol Urodyn 2016;35:980-6.

27. Abrams P. Bladder outlet obstruction index, bladder contractility index and bladder voiding efficiency: three simple indices to define bladder voiding function. BJU Int 1999;84:14-5.

28. Suh YS, Ko KJ, Kim TH, et al. Efficacy of Holmium Laser Transurethral Incision of the Prostate in Symptomatic Mild-to-Moderate Benign Prostate Enlargement Based on Preoperative Characteristics. Low Urin Tract Symptoms 2018;10:231-6.

29. Lee YJ, Lee JK, Kim JJ, et al. Development and validation of a clinical nomogram predicting bladder outlet obstruction via routine clinical parameters in men with refractory nonneurogenic lower urinary tract symptoms. Asian J Androl 2019;21:486-92. 